\title{
Anisotropic spline approximation with non-uniform B-splines
}

\author{
N. Sissouno**
}

September 5, 2016

\begin{abstract}
Recently the author and U. Reif introduced the concept of diversification of uniform tensor product B-splines. Based on this concept, we give a new constructive modification of non-uniform B-splines. The resulting spline spaces are perfectly fitted for the approximation of functions defined on domains $\Omega \subset \mathbb{R}^{2}$. We build a bounded quasiinterpolant and prove that for our spline spaces an anisotropic error estimate in the $L^{p}$ norm, $1 \leq p \leq \infty$, is valid. In particular, we show that the constant of the error estimate does not depend on the shape of $\Omega$ or the knot grid.
\end{abstract}

\section{Introduction}

B-splines are used in different fields of applications such as computer aided design and finite element analysis. In this context, lately, tensor product splines became important due to their good approximation properties over $\mathbb{R}^{d}$ or boxes and their efficient implementation [CHB09, HH13]. However, over arbitrary domains tensor product spline approximation cannot be used directly since some of those properties get lost. The concept of diversification of uniform tensor product B-splines over subsets $\Omega \subset \mathbb{R}^{d}$ is crucial for the construction [RS14] of a spline space such that an anisotropic error estimate with respect to the sup-norm can be obtained. Diversification itself is not connected to the uniformity of tensor product B-splines but the methods for proving the error estimate are tied to it. The aim of this paper is a generalisation of the results in [RS14] in two different ways. We adapt the construction to arbitrary knot sequences and, at the same time, consider the error with respect to the $L^{p}$-norm for $1 \leq p \leq \infty$.

For the spline space $\mathscr{S}_{n}(T, \Omega)$ of standard tensor product splines of coordinate order $n=\left(n_{1}, \ldots, n_{d}\right)$ with knots $T=\left(T_{1}, \ldots, T_{d}\right), T_{\sigma}=\left(\cdots \leq \tau_{\sigma,-1} \leq \tau_{\sigma, 0} \leq \tau_{\sigma, 1} \leq \ldots\right)$ with $\tau_{\sigma, k}<\tau_{\sigma, k+n_{\sigma}}, \sigma \in\{1, \ldots, d\}$, restricted to $\Omega$ [DDS80] show that

$$
\min _{s \in \mathscr{S}_{n}(T, \Omega)}\|f-s\|_{\Omega, L^{p}} \leq C \sum_{i=1}^{d} h_{i}^{n_{i}}\left\|\partial_{i}^{n_{i}} f\right\|_{\Omega, L^{p}}
$$

\footnotetext{
${ }^{* *}$ Corresponding author. Email: sissouno@ $@$ forwiss.uni-passau.de
} 
for some $C>0$, where $h=\left(h_{1}, \ldots, h_{d}\right)$ with $h_{\sigma}:=\max _{k}\left|\tau_{\sigma, k}-\tau_{\sigma, k+1}\right|$ is the maximal spacing of knots. As already stated in [RS14], the problem is that, on one hand, the set of appropriate domains is strongly restricted and, on the other hand, the constant $C$ is depending on the aspect ratio $\varrho:=\max _{i, j} h_{i} / h_{j}$. The latter problem appears in a similar fashion in [HR03] and [MR08].

It is hardly surprising that the spline space $\mathscr{S}_{n}(T, \Omega)$ is not the best choice for approximation on arbitrary subsets of $\mathbb{R}^{d}$. So, we construct a larger space $\mathscr{S}_{n}^{*}(T, \Omega)$ of non-uniform diversified splines and prove that estimate (1) is valid for bivariate approximations. It holds true for the same broad class of domains $\Omega \in \mathbb{R}^{2}$ as in the uniform case and the constant $C$ is independent of the aspect ratio.

The main differences between the uniform case [ $[\mathrm{RS14}]$ and the non-uniform case considered here are parts of the proof of the main result. Particularly, the concept of condensation and the construction of a quasi-interpolant as well as the proof of its boundedness are bound to the uniformity of the knots. We develop a new non-uniform condensation and give a construction of a quasi-interpolant for the resulting B-splines which is bounded in the $L^{p}$-norm for $1 \leq p \leq \infty$.

We present the univariate non-uniform condensation and some auxiliary univariate results in the second section.

In the third section, we introduce our main theorem claiming that (1) is true for approximation with non-uniform diversified B-splines. The section also contains necessary notions and the definition of the set of domains for which the theorem holds.

The main ingredients of the proof of the main theorem are non-uniform condensation, a local knot structure, and a quasi-interpolant. All those ingredients and the final proof are stated in the fourth section.

Although the key elements for the proof are new, the basic structure as well as diversification and the set of domains are the same as in the uniform case. Therefore, we will closely follow the structure and notation of [RS14]. For convenience of the reader, we will recall relevant material from [RS14] briefly, thus making our exposition self-contained.

\section{Issues in one variable}

In this section, the knot sequence is given by $T=\left\{\tau_{k}\right\}_{k \in \mathbb{Z}}$ with $\tau_{k} \leq \tau_{k+1}$ and $\tau_{k}<\tau_{k+n}$. As in the uniform case, this sequence is not a priori well adapted to a given domain $\omega=(\underline{\omega}, \bar{\omega})$ in the sense that $|\omega|:=\bar{\omega}-\underline{\omega}$ may be much smaller than the maximal knot spacing $h$. To adapt the knot sequence to the domain, two cases need to be considered:

1. If $|\omega| \leq h$, then the knot sequence is changed by replacing all knots outside of the domain by multiple knots at the boundary. The new knot sequence is given by $T^{\omega}:=$ $\left\{\tau_{k}^{\omega}\right\}_{k \in \mathbb{Z}}$, where

$$
\tau_{k}^{\omega}:= \begin{cases}\underline{\omega} & \text { for } k \in\left\{j \in \mathbb{Z}: \tau_{j} \leq \underline{\omega}\right\}, \\ \tau_{k} & \text { for } k \in\left\{j \in \mathbb{Z}: \tau_{j} \in \omega\right\}, \\ \bar{\omega} & \text { for } k \in\left\{j \in \mathbb{Z}: \tau_{j} \geq \bar{\omega}\right\} .\end{cases}
$$

2. If $|\omega|>h$, then nothing is changed, i.e., $T^{\omega}:=T$.

For example, given $\omega=(0,1)$ and $T$ with $h \geq 1$ and $\tau_{k} \notin \omega$ for all $k \in \mathbb{Z}$, the B-splines with respect to the knot sequence $T^{\omega}$ coincide with the Bernstein polynomials. Analogous to the 
uniform case, the process of replacing a given knot sequence $T$ by $T^{\omega}$ with maximal spacing $h^{\omega} \leq|\omega|$ under the condition that knots within the domain $\omega$ remain unchanged is called nonuniform condensation. It allows us to carry over some notations and properties from [RS14]: The non-uniform condensed $B$-splines with respect to $T^{\omega}$ are denoted by $b_{i}^{\omega}, i \in \mathbb{Z}$. The set of indices of active $B$-splines is given by $I_{\omega}:=\left\{i \in \mathbb{Z}: \operatorname{supp} b_{i} \cap \omega \neq \emptyset\right\}$, and we have $I_{\omega}=\left\{i \in \mathbb{Z}: \operatorname{supp} b_{i}^{\omega} \cap \omega \neq \emptyset\right\}$. The supports satisfy $\operatorname{supp} b_{i}^{\omega} \subset \operatorname{supp} b_{i}$ for $i \in I_{\omega}$ and the spanned spline spaces coincide, i.e. $\operatorname{span}\left\{b_{i} \chi(\omega): i \in I_{\omega}\right\}=\operatorname{span}\left\{b_{i}^{\omega} \chi(\omega): i \in I_{\omega}\right\}$, where $\chi(\omega)$ denotes the characteristic function of the domain.

The construction of a quasi-interpolant, which is necessary for the proof of the main theorem, consists of two steps. First, a projection into the space of polynomials and, second, a representation of polynomials in terms of splines. We will briefly recall some univariate results, which will be of use for this construction.

Let $I^{*} \subseteq I$ be intervals in $\mathbb{R}$ and $\left\{\ell_{\alpha} \in L^{1}(I): \alpha<n\right\}$ the normalised Legendre polynomials of degree $<n$ over $I$. A projection of functions $f \in L^{1}\left(I^{*}\right)$ onto the space of polynomials is then given by

$$
\hat{f}=\sum_{\alpha=0}^{n-1}\left\langle f, \ell_{\alpha}\right\rangle \ell_{\alpha} .
$$

For estimates in the $L^{p}$-norm the following results will be useful.

Lemma 1. If the Legendre polynomials $\left\{\ell_{\alpha} \in L^{1}(I): \alpha<n\right\}$ for $I \subset \mathbb{R}$ of size $|I|=h$, $h>0$, are normalised such that

$$
\int_{I} \ell_{\alpha} \ell_{\beta}=\left\{\begin{array}{ll}
0 & \alpha \neq \beta \\
1 & \alpha=\beta
\end{array},\right.
$$

then

$$
\left\|\ell_{\alpha}\right\|_{I, p}\left\|\ell_{\alpha}\right\|_{I, \infty} \leq c_{n} h^{\frac{1}{p}-1}
$$

with a constant only depending on $n$.

Proof. Without loss of generality, we assume that $I=[0, h]$. Let $L_{\alpha}, \alpha<n$, denote the normalised Legendre polynomials over $[-1,1]$. Then one has $\ell_{\alpha}(x)=\sqrt{\frac{2}{h}} L_{\alpha}\left(\frac{2 x-h}{h}\right)$. Since $L_{\alpha}$ is bounded by a constant only depending on $n$, we get

$$
\left\|\ell_{\alpha}\right\|_{I, \infty} \leq c_{n}^{\prime} h^{-\frac{1}{2}}
$$

Therefore, we have

$$
\left\|\ell_{\alpha}\right\|_{I, p}\left\|\ell_{\alpha}\right\|_{I, \infty} \leq\|1\|_{I, p}\left\|\ell_{\alpha}\right\|_{I, \infty}^{2} \leq c_{n} h^{\frac{1}{p}-1}
$$

with $c_{n}:=\left(c_{n}^{\prime}\right)^{2}$.

The representation of polynomials in terms of non-uniform splines is slightly different from the representation in the uniform case. For a univariate B-spline $b_{i}$, let $I_{i}:=\left[\tau_{j}, \tau_{j+1}\right)$ with $j \in\{i, \ldots, i+n-1\}$ and $\left|I_{i}\right| \geq \frac{1}{n}\left|\operatorname{supp} b_{i}\right|$ be the longest knot interval of $\operatorname{supp} b_{i}$. For any polynomial $p \in \mathbb{P}_{n}$ of order $n$, the coefficient $s_{i}$ in the representation $p=\sum_{i} b_{i} s_{i}$ can be determined as linear combination of the values of $p$ at the points $\lambda_{i, m}=\tau_{j}+\frac{m-1}{n-1}\left|I_{i}\right|$, $m=1, \ldots, n$, and certain weights $w_{i, 1}^{n}, \ldots, w_{i, n}^{n}$ that can be computed [HH13] by solving the linear system $\sum_{m=1}^{n} w_{i, m}^{n}(m-l)^{n-1}=\frac{(n-1)^{n-1}}{\left|I_{i}\right|^{n-1}} \psi_{i}^{n}\left(\lambda_{i, l}\right), l=1, \ldots, n$, where $\psi_{i}^{n}(\tau):=$ 
$\left(\tau_{i+1}-\tau\right) \cdots\left(\tau_{i+n-1}-\tau\right)$. Since $\left|\psi_{i}^{n}\left(\lambda_{i, l}\right)\right| \leq\left|\operatorname{supp} b_{i}\right|^{n-1}$, the right hand side is bounded by $n^{2 n}$. Additionally, the entries $(m-l)^{n-1}$ do not depend on $T$. Therefore, the weights $w_{i, m}^{n}$ are bounded by a constant only depending on $n$ and independent of $T$ and $b_{i}$. Now, we have

$$
p(x)=\sum_{i \in I_{\omega}} b_{i}(x) \sum_{m=1}^{n} w_{i, m}^{n} p\left(\lambda_{i, m}\right), \quad x \in \omega .
$$

\section{Bivariate approximation}

Adapting univariate condensation to the bivariate case does not solve the problem that the constant in (1) depends on the aspect ratio $\varrho:=\max _{i, j} h_{i} / h_{j}$. The dependence is caused by B-splines whose intersection with $\Omega$ consists of several connected components (see [RS14, Section 2.2] for an example). This problem can be solved by the concept of diversification, which will be explained in this section and enables us to present the main theorem.

At first we need some notations and definitions recalling relevant material from [ [RS14].

\subsection{Notation}

Let $\Omega, M$ and $A$ denote subsets of $\mathbb{R}^{2}$ and $I$ an interval in $\mathbb{R}^{2}$ given by the Cartesian product of two intervals in $\mathbb{R}$ with size $|I|:=\sup \{x-y: x, y \in I\}$, where the supremum is understood component-wise. The size of a subset $M$ is defined as the size of its bounding box, i.e., $|M|:=$ $|\mathcal{B}(M)|$ for bounding box $\mathcal{B}(M)=\mathcal{B}_{1}(M) \times \mathcal{B}_{2}(M):=\inf _{|I|}\{I: M \subset I\}$. Given $h \in[0, \infty]^{2}$ we define the $h$-neighbourhood of $M$ by $\mathcal{N}(M, h):=\left\{x \in \mathbb{R}^{2}:|\{x\} \cup M| \leq|M|+h\right\}$. The set of connected components of $M \cap \Omega$ is denoted by $\mathcal{C}_{\Omega}(M)$. If, in addition, $M$ is connected and $M \subset \Omega$, we need two more notations, namely, for the elements of $\mathcal{C}_{\Omega}(\mathcal{B}(M))$ and $\mathcal{C}_{\Omega}(\mathcal{N}(M, h))$ which contain $M$, i.e., the pruned bounding box $\mathcal{B}_{\Omega}(M):=\left\{A \in \mathcal{C}_{\Omega}(\mathcal{B}(M))\right.$ : $M \subseteq A\}$ and the local h-neighbourhood $\mathcal{N}_{\Omega}(M, h):=\left\{A \in \mathcal{C}_{\Omega}(\mathcal{N}(M, h)): M \subseteq A\right\}$.

From now on, the index $\sigma \in\{1,2\}$ is always addressing the two coordinate directions, and the components of two-dimensional objects are tagged by the prepending subscript $\sigma$. For instance, $x=\left(x_{1}, x_{2}\right) \in \mathbb{R}^{2}$ or $x_{j}=\left(x_{1, j}, x_{2, j}\right)$ for a sequence $\left\{x_{j}\right\}_{j}$ in $\mathbb{R}^{2}$.

We give some further notations connected to the spline space $\mathscr{S}_{n}(T, \Omega)$ spanned by the tensor product B-splines $B_{i}(x):=b_{1, i_{1}}\left(x_{1}\right) b_{2, i_{2}}\left(x_{2}\right)$ of coordinate order $n \in \mathbb{N}^{2}$ for $\left.x \in \mathbb{R}^{2}\right|_{\Omega}$ and $i \in \mathbb{Z}^{2}$. Here, $b_{\sigma, i_{\sigma}}$ denotes the univariate B-splines of order $n_{\sigma}$ with knots $T_{\sigma}$. We need individual knots $\tau_{k}:=\left(\tau_{1, k}, \tau_{2, k}\right), k \in \mathbb{Z}^{2}$, with midpoints $\mu_{k}:=\left(\tau_{k-(1,1)}+\tau_{k}\right) / 2$ and the width of a cell $h_{k}:=\left|\tau_{k}-\tau_{k-(1,1)}\right|$. A grid cell $\Gamma_{k}$ is then defined by the $h_{k} / 2$ neighbourhood of $\mu_{k}$, that is, $\Gamma_{k}:=\mathcal{N}\left(\left\{\mu_{k}\right\}, h_{k} / 2\right)$. The support of a B-spline is denoted by $S_{i}:=\operatorname{supp} B_{i}=\mathcal{B}\left(\left\{\tau_{i}\right\} \cup\left\{\tau_{i+n}\right\}\right)$. Further, we define $\bar{n}:=\max \left(n_{1}, n_{2}\right)$.

\subsection{Main result}

As already mentioned in the introduction, diversification as well as the set of domains are the same as in the uniform case. Therefore, we just state the definitions and a few important comments (see [RS14] for more details) before we present the main result.

Definition 1. Let $\Phi=[a, \varphi]$ be a pair consisting of the real number $a>0$ and a continuous function $\varphi: X \rightarrow \mathbb{R}_{>0}$ defined on the interval $X:=[-a, a]$. With $X^{\delta}:=[-a+\delta, a-\delta]$ the 
sub-interval with margin $\delta \in \mathbb{R}$, let

$$
\Phi^{\delta}:=\left\{x \in X^{\delta} \times \mathbb{R}: \delta<x_{2}<\varphi\left(x_{1}\right)\right\} .
$$

An axis-aligned isometry in $\mathbb{R}^{2}$ is a composition of a translation and a rotation by an integer multiple of $\pi / 2$.

A subset $\Omega \subset \mathbb{R}^{2}$ is called a finite (or bounded) graph domain with parameter $h_{0} \in \mathbb{R}_{>0}$ if there exists an finite index set $R \subset \mathbb{N}$, axis-aligned isometries $\Sigma_{r}$, and pairs $\Phi_{r}=\left[a_{r}, \varphi_{r}\right]$ as above with $a_{r}>h_{0}$ and $\min _{X_{r}} \varphi_{r}>h_{0}$ such that

$$
\Omega=\bigcup_{r \in R} \Sigma_{r}\left(\Phi_{r}^{\delta}\right), \quad 0 \leq \delta \leq h_{0}
$$

Basically, those domains consist of the union of subdomains such that the boundary is piecewise represented by graphs of continuous functions and $h_{0}$ guarantees a certain amount of overlap of $\Sigma_{r}\left(\Phi_{r}^{0}\right)$. This overlap allows the following argument, which will be used repeatedly, and without further notice: Given $\Omega$ as above, let $M \subset \Omega$ be an arbitrary subset of size $|M| \leq 2 h_{0}$. There exists a point $x \in M$ satisfying $M \subset \mathcal{N}_{\Omega}\left(\{x\}, h_{0}\right)$ and an index $r \in R$ such that $x \in \Sigma_{r}\left(\Phi_{r}^{h_{0}}\right)$. Hence, $M \subset \Sigma_{r}\left(\Phi_{r}^{0}\right)$. Without loss of generality, we can assume that $\Sigma_{r}$ is the identity. Since the value of the index $r \in R$ will be irrelevant, we drop the index $r$ and write $\Phi=[a, \varphi]$ instead of $\Phi_{r}=\left[a_{r}, \varphi_{r}\right]$ when examining $M$. In particular, it is $M \subset \Phi^{0}$.

The idea of diversification is to use a copy of a given B-spline $B_{i}$ for each connected component of its support in $\Omega$, i.e., the diversified $B$-splines are defined by

$$
B_{j}:=B_{i} \chi(\gamma), \quad j=(i, \gamma) \in J:=\left\{(i, \gamma): i \in \mathbb{Z}^{2}, \gamma \in \mathcal{C}_{\Omega}\left(S_{i}\right)\right\},
$$

with support $S_{j}:=\operatorname{supp} B_{j}=\operatorname{supp} \chi(\gamma)$, see Figures 1(a) and 1(b). From now on, the subscripts $i \in \mathbb{Z}^{2}$ indicate standard B-splines, while subscripts $j=(i, \gamma) \in J$ indicate their diversified descendants. The diversified B-splines span the space $\mathscr{S}_{n}^{*}(T, \Omega)$ and we have $\mathscr{S}_{n}(T, \Omega) \subseteq \mathscr{S}_{n}^{*}(T, \Omega)$.

To formulate our main result, we define the anisotropic Sobolev space $W_{p}^{n}(\Omega), 1 \leq p \leq \infty$ of order $n \in \mathbb{N}^{2}$ as the space of real-valued functions $f \in L^{p}(\Omega)$ with partial derivatives $\partial_{1}^{n_{1}} f, \partial_{2}^{n_{2}} f \in L^{p}(\Omega)$.

Theorem 1. Let $\Omega$ be a graph domain with parameter $h_{0}$, and let $n \in \mathbb{N}^{2}$. There exists a constant $C$ depending only on $n$ and $p$ such that

$$
\inf _{s \in \mathscr{S}_{n}^{*}(T, \Omega)}\|f-s\|_{\Omega, p} \leq C\left(h_{1}^{n_{1}}\left\|\partial_{1}^{n_{1}} f\right\|_{\Omega, p}+h_{2}^{n_{2}}\left\|\partial_{2}^{n_{2}} f\right\|_{\Omega, p}\right)
$$

for any $f \in W_{p}^{n}(\Omega), 1 \leq p \leq \infty$, and any knot sequence $T$ with grid width $h \leq h_{0} /(\bar{n}+1)$.

\section{Proof}

The key element of the proof is the construction of a bounded quasi-interpolant, which is given in Section 4.4. The necessary structure is provided by non-uniform condensation as described in the first subsection. The method of transferring the univariate condensation into two dimensions is originated in the uniform condensation described in [RS14]. 


\subsection{Condensation}

We describe how to apply condensation to diversified B-splines. The process of condensation is not applied directly with respect to the complete $\Omega$. It is applied locally to subdomains of $\Omega$ which are extensions of the supports $S_{j}$ and are determined by horizontally and vertically unbounded strips or intervals depending on the supports $S_{i}$. Given the index $i \in \mathbb{Z}^{2}$, we define the unbounded extensions of the support $S_{i}$ in $x_{1}$ - and $x_{2}$-direction as the unbounded intervals $W_{1, i}:=\mathcal{N}\left(S_{i},(\infty, 0)\right)$ and $W_{2, i}:=\mathcal{N}\left(S_{i},(0, \infty)\right)$. Further, for $j=(i, \gamma) \in J$ we denote the connected components of $W_{\sigma, i}$ containing the support $S_{j}$ by $W_{\sigma, j}:=\left\{A \in \mathcal{C}_{\Omega}\left(W_{\sigma, i}\right): S_{j} \subset\right.$ $A\}$. See Figures 1(c) and 1(d) for examples of these sets. Now, condensation, as described in Section 2, is conducted with respect to the univariate intervals $\omega_{\sigma, j}:=\mathcal{B}_{\sigma}\left(W_{\sigma, j}\right)$ and we obtain the non-uniform condensed diversified B-splines (or briefly cdB-splines)

$$
B_{j}^{*}(x):=b_{1, i_{1}}^{\omega_{1, j}}\left(x_{1}\right) b_{2, i_{2}}^{\omega_{2, j}}\left(x_{2}\right) \chi(\gamma), \quad j=(i, \gamma) \in J .
$$

For their supports $S_{j}^{*}:=\operatorname{supp} B_{j}^{*}$ we have $S_{j}^{*}=S_{j} \subset S_{i}$ and $\left|S_{j}^{*}\right|=\left|S_{j}\right| \leq \bar{n} h<h_{0}$. The supports of non-uniform cdB-splines are illustrated in Figures 1(e) and 1(f).

We give some further notations related to the cdB-splines following the notations of tensor product B-splines given in Section 3.1. We use $T_{j}^{*}=\left(T_{1, j}^{*}, T_{2, j}^{*}\right)$ with $T_{\sigma, j}^{*}=T_{\sigma}^{\omega_{\sigma, j}}$ and maximal grid width $h_{j}^{*}$ for the condensed knot sequences, $\tau_{j, k}^{*}=\left(\tau_{1, j, k}^{*}, \tau_{2, j, k}^{*}\right)$ with $\tau_{\sigma, j, k}^{*}=$ $\tau_{\sigma, k}^{\omega_{\sigma, j}}$ for the individual knots, and $\mu_{j, k}^{*}=\left(\tau_{j, k-(1,1)}^{*}+\tau_{j, k}^{*}\right) / 2$ for the midpoints. The local width of a cell is denoted by $h_{j, k}^{*}$.

\subsection{Local knot structure}

Locally, the condensed knot sequences $T_{j}^{*}$ still have a structure such that some important properties of the spline space, like partition of unity, remain unchanged. In this subsection, we introduce some of those properties and the main arguments for their validity.

To analyse the structure of knot sequences in a proximity of a grid cell $\Gamma_{k}$, we denote the connected components of $\Gamma_{k} \cap \Omega$ by $\Gamma_{\ell}, \ell \in L:=\left\{(k, \gamma): k \in \mathbb{Z}^{2}, \gamma \in \mathcal{C}_{\Omega}\left(\Gamma_{k}\right)\right\}$. We have $\Omega=\bigcup_{\ell \in L} \Gamma_{\ell}$. Given $\ell \in L$, the neighbourhood of those cdB-splines which are relevant for $\Gamma_{\ell}$ is given by

$$
\Gamma_{\ell}^{*}:=\bigcup_{j \in J_{\ell}} S_{j}^{*} \quad \text { with } J_{\ell}:=\left\{j \in J: S_{j}^{*} \cap \Gamma_{\ell} \neq \emptyset\right\} .
$$

Since $\left|\Gamma_{\ell}^{*}\right| \leq(2 \bar{n}-1) h \leq 2 h_{0}$, recalling the arguments below Definition 1 , we can assume that $\Gamma_{\ell}^{*} \subset \Phi^{0}$. Therefore, for any $j \in J_{\ell}$, we have $\left|\omega_{2, j}\right| \geq h_{0}$ and, thus, condensation leaves the knot sequence in $x_{2}$-direction as it is and we get $T_{2, j}^{*}=T_{2}$. In $x_{1}$-direction there may be a modification which is the same for all diversified B-splines with equal index $i_{2}$. More precisely, for all $j \in J_{\ell}^{i_{2}}:=\left\{j^{\prime}=\left(i^{\prime}, \gamma^{\prime}\right) \in J_{\ell}: i_{2}^{\prime}=i_{2}\right\}$ there exists an interval $\omega^{\prime}$ such that we get $T_{1, j}^{*}:=T_{1}^{\omega_{1, j}}=T_{1}^{\omega^{\prime}}$ and $b_{1, j}:=b_{1, i_{1}}^{\omega_{1, j}}=b_{1, i_{1}}^{\omega^{\prime}}$. Those knot sequences depend only on the component $i_{2}$ of the index $j=(i, \gamma) \in J_{\ell}^{i_{2}}$. For a proof, we refer to [RS14]. Together, this allows us to write any spline $s \in \mathscr{S}_{n}^{*}(T, \Omega)$ locally as

$$
s(x)=\sum_{j \in J_{\ell}} s_{j} B_{j}^{*}(x)=\sum_{i_{2} \in \mathbb{Z}} b_{2, i_{2}}\left(x_{2}\right) \sum_{j \in J_{\ell}^{i_{2}}} s_{j} b_{1, j}\left(x_{1}\right), \quad x \in \Gamma_{\ell} .
$$


As mentioned above, due to the local structure, cdB-splines form a partition of unity, i.e., if $s_{j}=1$ for all $j \in J_{\ell}$ in (2), then

$$
\sum_{j \in J_{\ell}} B_{j}^{*}(x)=\sum_{i_{2} \in \mathbb{Z}} b_{2, i_{2}}\left(x_{2}\right) \sum_{j \in J_{\ell}^{i_{2}}} b_{1, j}\left(x_{1}\right)=\sum_{i_{2} \in \mathbb{Z}} b_{2, i_{2}}\left(x_{2}\right)=1, \quad x \in \Gamma_{\ell} .
$$

Hence, using $\left|\Gamma_{\ell}\right| \leq h_{j}^{*}$ for $j \in J_{\ell}$, it follows that

$$
\left\|B_{j}^{*}\right\|_{\Gamma_{\ell}, p}^{p} \leq h_{1, j}^{*} h_{2, j}^{*}
$$

\subsection{Representation of polynomials}

As already mentioned in the preceding section, the construction of a quasi-interpolant for the proof consists of a projection onto the space of polynomials combined with the representation of polynomials in terms of splines, particularly cdB-splines. The latter will be described in this section.

We consider polynomials $p \in \mathbb{P}_{n}$ of coordinate order $n \in \mathbb{N}^{2}$. The support of the unrestricted cdB-spline corresponding to $B_{j}^{*}$ is given by $S_{j}^{\prime}:=\mathcal{B}\left(\left\{\tau_{j, i}^{*}\right\} \cup\left\{\tau_{j, i+n}^{*}\right\}\right)$, see Figures 1(e) and 1(f), Because of the tensor product structure, the representation can be build analogous to the univariate case described in Section 2. We set $\Gamma_{j, K}^{*}:=\mathcal{N}\left(\left\{\mu_{j, K}^{*}\right\}, h_{j, K}^{*} / 2\right)$ for $K_{\sigma} \in\left\{i_{\sigma}+1, \ldots, i_{\sigma}+n_{\sigma}\right\}$ with $\left|\Gamma_{j, K}^{*}\right| \geq \frac{1}{n}\left|S_{j}^{\prime}\right|$ for the largest subcell of $S_{j}^{\prime}$. Then we define the linear functional $P_{j}: \mathbb{P}_{n} \rightarrow \mathbb{R}$ by

$$
P_{j} p:=\sum_{m_{1}=1}^{n_{1}} \sum_{m_{2}=1}^{n_{2}} w_{j, m_{1}}^{n_{1}} w_{j, m_{2}}^{n_{2}} p\left(\lambda_{1, j, m}^{*}, \lambda_{2, j, m}^{*}\right), \quad j=(i, \gamma),
$$

with $\lambda_{\sigma, j, m}^{*}=\tau_{\sigma, j, K-(1,1)}^{*}+\frac{m_{\sigma}-1}{n_{\sigma}-1} \cdot\left|\tau_{\sigma, j, K-(1,1)}^{*}-\tau_{\sigma, j, K}^{*}\right|$. We claim that

$$
p(x)=\sum_{j \in J} B_{j}^{*}(x) P_{j} p, \quad x \in \Omega .
$$

Since $T_{2, j}^{*}=T_{2}$, we have $\left(\lambda_{2, j, m}^{*}\right)^{d_{2}}=\lambda_{2, i, m}^{d_{2}}$, and $w_{j, m_{2}}^{n_{2}}=w_{i_{2}, m_{2}}^{n_{2}}$ is independent of $j$. Using this, the proof of (5) is straightforward considering any monomial $p^{d}(x):=x_{1}^{d_{1}} x_{2}^{d_{2}}$ of coordinate degree $d<n$ with $x \in \Gamma_{\ell}$ for an arbitrary index $\ell \in L$.

\subsection{Quasi-interpolation}

In this section we construct a suitable quasi-interpolant. We will use the representation of polynomials developed in the previous subsection. Since the evaluation points $\lambda_{j, m}^{*}$ of $P_{j}$ may lie outside of $\Omega$, we first need a local approximation.

For any $j \in J$, we denote by $S_{j}^{+}:=\mathcal{N}_{\Omega}\left(S_{j}^{*}, h_{j}^{*}\right)$ the local $h_{j}^{*}$-neighbourhood of $S_{j}^{*}$. Then, in the same way as in the uniform case, there exists an interval $H_{j}^{*} \subset S_{j}^{+}$of size $\left|H_{j}^{*}\right|=h_{j}^{*}$. The interval as well as the local $h_{j}^{*}$-neighbourhood are illustrated in Figures $1(\mathrm{~g})$ - $1(\mathrm{j})$. The interval is given by $H_{j}^{*}:=\omega^{\prime} \times\left[\tau_{2, i}-h_{2, j}^{*}, \tau_{2, i}\right]$, where $\omega^{\prime} \subset \omega_{1, j}$ with length $\left|h_{1, j}^{*}\right|$ containing some point $x=\left(x_{1}, \tau_{2, i}\right) \in S_{j}^{*}$ on the lower bound of $S_{j}^{*}$. For more details see [RS14]. Next, we define the local approximation over $H_{j}^{*}$ as the linear operator $A_{j}: W_{p}^{n}\left(H_{j}^{*}\right) \rightarrow \mathbb{P}_{n}$ defined by

$$
A_{j} f:=\sum_{\alpha<n}\left\langle f, \ell_{\alpha, j}\right\rangle \ell_{\alpha, j}
$$


where $\left\{\ell_{\alpha, j} \in L^{1}\left(H_{j}^{+}\right): \alpha<n\right\}$ are the normalised tensor product Legendre polynomials over $H_{j}^{+}:=\mathcal{N}\left(H_{j}^{*}, \bar{n} h_{j}^{*}\right)$. So, $A_{j}$ is mapping the function $f$ to the polynomial which is best approximating on the interval $H_{j}^{*}$ with respect to the $L^{2}$-norm.

Now, we can define the quasi-interpolant $Q: W_{p}^{n}(\Omega) \rightarrow \mathscr{S}_{n}^{*}(T, \Omega)$ by

$$
Q f:=\sum_{j \in J} B_{j}^{*} Q_{j} f, \quad Q_{j}:=P_{j} A_{j} .
$$

The operator $Q$ reproduces polynomials $p \in \mathbb{P}_{n}$, what follows immediately from (5). Further, the functionals $Q_{j}: W_{p}^{n}\left(H_{j}^{*}\right) \rightarrow \mathbb{R}, j \in J$, can be written as $Q_{j} f=\left\langle f, q_{j}\right\rangle$, which is the inner product of $f$ with the polynomial

$$
q_{j}:=\sum_{m_{1}=1}^{n_{1}} \sum_{m_{2}=1}^{n_{2}} \sum_{\alpha<n} w_{j, m_{1}}^{n_{1}} w_{j, m_{2}}^{n_{2}} \ell_{\alpha, j}\left(\lambda_{1, j, m}^{*}, \lambda_{2, j, m}^{*}\right) \cdot \ell_{\alpha, j} .
$$

Since $\lambda_{j, m}^{*} \in S_{j}^{\prime} \subset H_{j}^{+}$and, as already remarked in Section 2. all $w_{j, m_{i}}^{n_{i}}, i=1,2$, are bounded by a constant only depending on $n$, we get

$$
\left\|q_{j}\right\|_{H_{j}^{*}, q} \leq \tilde{c}_{n} \sum_{\alpha<n}\left\|\ell_{\alpha, j}\right\|_{H_{j}^{+}, \infty}\left\|\ell_{\alpha, j}\right\|_{H_{j}^{+}, q} \leq c_{n}^{\prime}\left(h_{1, j}^{*} h_{2, j}^{*}\right)^{\frac{1}{q}-1}
$$

where we used Lemma 1 and $c_{n}^{\prime}:=\tilde{c}_{n} \cdot c_{n}$. Therefore, using Hölder's inequality, we see that the functionals $Q_{j}$ are bounded by

$$
\left|Q_{j} f\right|=\left|\left\langle f, q_{j}\right\rangle\right| \leq c_{n}^{\prime}\left(h_{1, j}^{*} h_{2, j}^{*}\right)^{\frac{1}{q}-1}\|f\|_{H_{j}^{*}, p} .
$$

Hence, for $p=\infty$ and $q=1$ the boundedness of the spline $Q f$ on $\Gamma_{\ell}$ follows directly, since, using (3),

$$
\|Q f\|_{\Gamma_{\ell}, \infty} \leq \max _{j \in J_{\ell}}\left|Q_{j} f\right| \leq c_{n}^{\prime} \max _{j \in J_{\ell}}\|f\|_{H_{j}^{*}, \infty} .
$$

Applying Minkowski's inequality and (4) as well as (6) in the case of $p<\infty$, we have

$$
\|Q f\|_{\Gamma_{\ell}, p} \leq \sum_{j \in J_{\ell}}\left\|B_{j}^{*} Q_{j} f\right\|_{\Gamma_{\ell}, p}=\sum_{j \in J_{\ell}}\left|Q_{j} f\right|\left\|B_{j}^{*}\right\|_{\Gamma_{\ell}, p} \leq c_{n}^{\prime} \sum_{j \in J_{\ell}}\|f\|_{H_{j}^{*}, p} \leq \bar{n}^{2} c_{n}^{\prime} \max _{j \in J_{\ell}}\|f\|_{H_{j}^{*}, p}
$$

where we use in the last inequality that the number of elements in $J_{\ell}$ is $n_{1} \cdot n_{2} \leq \bar{n}^{2}$.

In summary, a bound of the spline $Q f$ on $\Gamma_{\ell}$ for $1 \leq p \leq \infty$ is given by

$$
\|Q f\|_{\Gamma_{\ell}, p} \leq c_{n, p} \max _{j \in J_{\ell}}\|f\|_{H_{j}^{*}, p}
$$

with $c_{n, p}:=c_{n}^{\prime}$ for $p=\infty$ and $c_{n, p}:=\bar{n}^{2} c_{n}^{\prime}$ else.

\subsection{Error estimate}

To prove Theorem 1 for $f \in W_{p}^{n}(\Omega)$, we first consider the approximation $s:=Q f$ on an arbitrary grid cell $\Gamma_{\ell}, \ell \in L$. We define

$$
\Gamma_{\ell}^{+}:=\mathcal{B}_{\Omega}\left(\bigcup_{j \in J_{\ell}} S_{j}^{+}\right)
$$


It contains all parts of $\Omega$ with potential influence on $\left.s\right|_{\Gamma_{\ell}}$. In particular, $H_{j}^{*} \subset \Gamma_{\ell}^{+}$for all $j \in J_{\ell}$. We can assume that $\Gamma_{\ell}^{+} \in \Phi^{0}$ since $\left|\Gamma_{\ell}^{+}\right| \leq(2 \bar{n}+1) h \leq 2 h_{0}$.

In [RS14] the construction of a set $\Gamma$ with $\Gamma_{\ell}^{+} \subset \Gamma \subset \Phi^{0}$ and $|\Gamma| \leq(2 \bar{n}+2) h$ is shown. Since this construction can be transfered without any changes, according to [Rei12], Theorem 2.5 , there exists a polynomial $p \in \mathbb{P}_{n}$ such that the error $\Delta:=f-p$ satisfies

$$
\|\Delta\|_{\Gamma, p} \leq c_{n, p}^{*}\left(h_{1}^{n_{1}}\left\|\partial_{1}^{n_{1}} f\right\|_{\Omega, p}+h_{2}^{n_{2}}\left\|\partial_{2}^{n_{2}} f\right\|_{\Omega, p}\right),
$$

where the constant $c_{n, p}^{*}$ depends only on $n$ and $p$. Eventually, we use reproduction of polynomials by the quasi-interpolant $Q$ and (7) to obtain

$$
\begin{aligned}
\|f-Q f\|_{\Gamma_{\ell}, p} & \leq\|\Delta\|_{\Gamma_{\ell, p}}+\|Q \Delta\|_{\Gamma_{\ell}, p} \leq\|\Delta\|_{\Gamma_{\ell}, p}+c_{n, p} \max _{j \in J_{\ell}}\|\Delta\|_{H_{j}^{*}, p} \\
& \leq\left(1+c_{n, p}\right)\|\Delta\|_{\Gamma, p} \leq\left(1+c_{n}\right) c_{n, p}^{*}\left(h_{1}^{n_{1}}\left\|\partial_{1}^{n_{1}} f\right\|_{\Omega, p}+h_{2}^{n_{2}}\left\|\partial_{2}^{n_{2}} f\right\|_{\Omega, p}\right) .
\end{aligned}
$$

The index $\ell \in L$ was chosen arbitrarily and, on the other hand, $|\Gamma| \leq(2 \bar{n}+1) h$ for all $\ell \in L$. Therefore, those restricted intervals can only overlap $\tilde{c} \cdot \bar{n}^{2}$ times, and the claim of Theorem 1 follows with

$$
C:= \begin{cases}\left(1+c_{n}^{\prime}\right) c_{n, p}^{*} & \text { for } p=\infty \\ \left(\bar{n}^{2}+\bar{n}^{4} c_{n}^{\prime}\right) c_{n, p}^{*} \tilde{c} & \text { else. }\end{cases}
$$

\section{Conclusion}

In this paper we generalised the ideas presented in [RS14] to arbitrary knot sequences and to error estimates with respect to anisotropic Sobolev norms. In comparison to the uniform theory the following conclusions can be made:

- In the bivariate case, diversification of tensor product B-splines provides a construction of spline spaces which have optimal approximation properties not only for uniform knot sequences but also for non-uniform knot sequences.

- Non-uniform condensation is used for the construction of bounded quasi-interpolants for arbitrary tensor product spline spaces. Since non-uniform condensation, as described in Section 2, is only depending on the size of the domain and is independent of the number of knots inside of the domain, it is even easier than the condensation described in [RS14].

- In three or more variables, diversification can be used for spline spaces with arbitrary knot sequences, but as already shown in [RS14] even for convex domains with smooth boundary the constant of the error estimate can depend on the mesh ratio. In the case of convex domains diversification leaves the spline space as it is and (non-uniform) condensation is just a change of basis, therefore the dependence remains unaffected.

An open question for future research is how the constants of error estimates for spline approximation are connected to the geometry of domains in higher dimensions. 


\section{References}

[CHB09] J. A. Cottrell, T. J. R. Hughes, and Y. Bazilevs. Isogeometric Analysis: Toward Integration of CAD and FEA. John Wiley \& Sons, 2009.

[DDS80] W. Dahmen, R. De Vore, and K. Scherer. Multi-dimensional spline approximation. SIAM J. Numer. Anal., 17(3):380-402, 1980.

[HH13] K. Höllig and J. Hörner. Approximation and modeling with B-splines. SIAM, 2013.

[HR03] K. Höllig and U. Reif. Nonuniform WEB-Splines. Computer Aided Geometric Design, 20(5):277-294, 2003.

[MR08] B. Mößner and U. Reif. Stability of tensor product B-splines on domains. Journal of Approximation Theory, 154(1):1-19, 2008.

[Rei12] U. Reif. Polynomial approximation on domains bounded by diffeomorphic images of graphs. Journal of Approximation Theory, 164:954-970, 2012.

[RS14] U. Reif and N. Sissouno. Approximation with Diversified B-Splines. CAGD, 31(78):510-520, 2014 


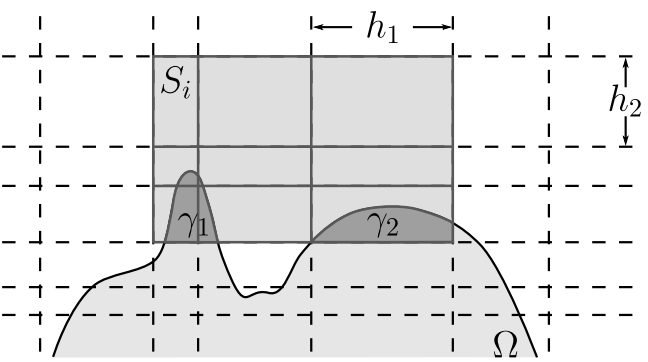

(a) Support of $B_{i}$

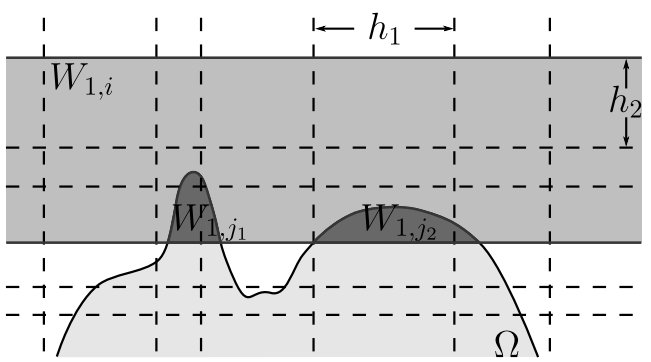

(c) Extension of $S_{i}$ in $x_{1}$-direction

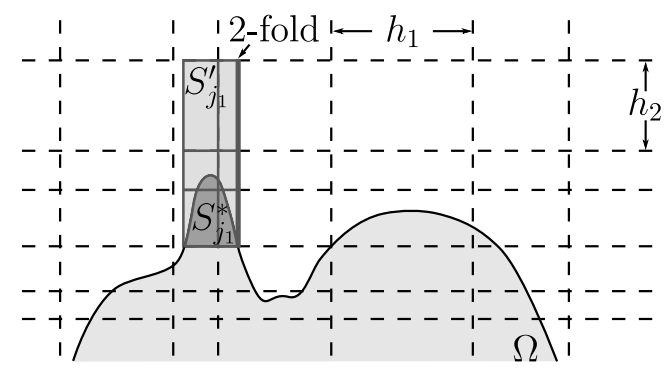

(e) Support of (unrestricted) cdB-spline $B_{j_{1}}^{*}$

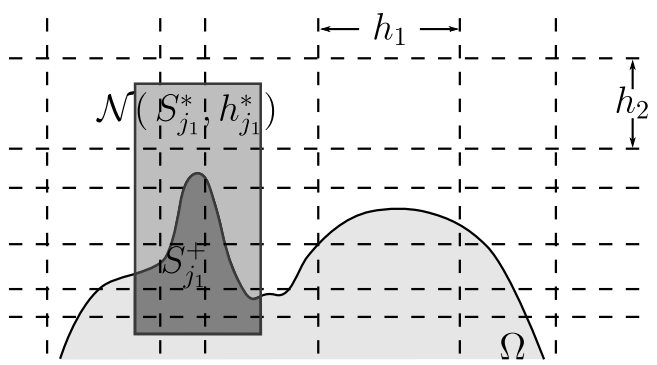

(g) Neighbourhood of $S_{j_{1}}^{*}$

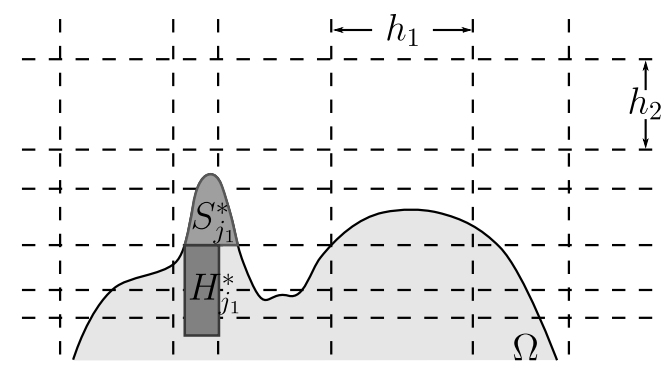

(i) Domain $H_{j_{1}}^{*}$ for local approximation

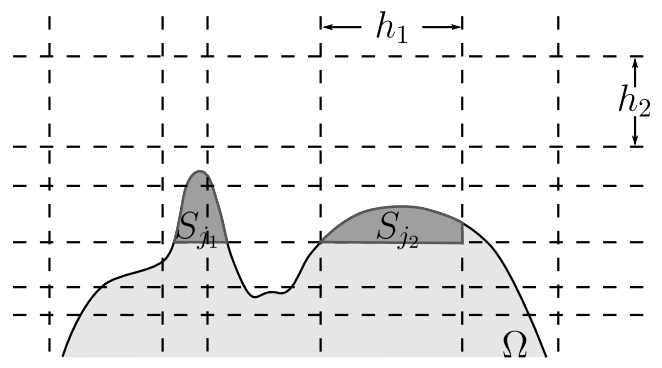

(b) Supports of diversified B-splines

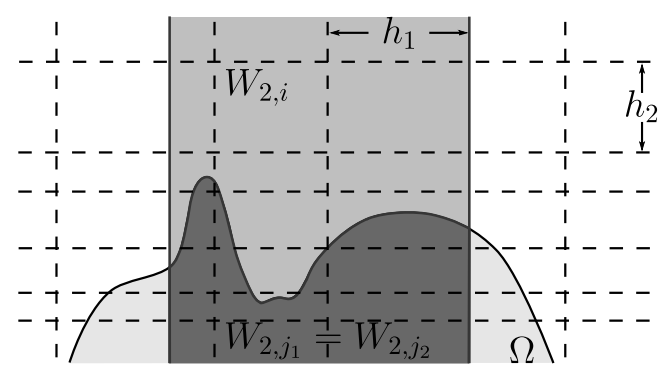

(d) Extension of $S_{i}$ in $x_{2}$-direction

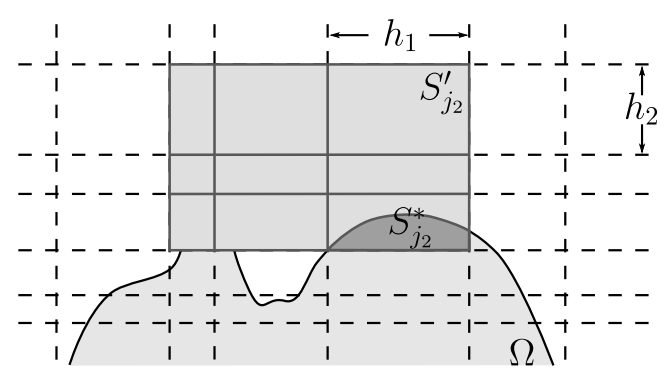

(f) Support of (unrestricted) cdB-spline $B_{j_{2}}^{*}$

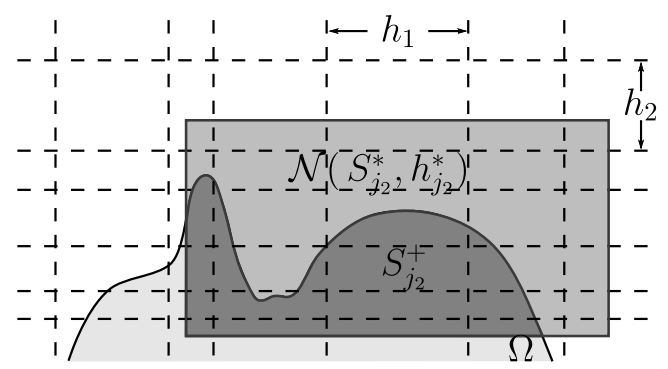

(h) Neighbourhood of $S_{j_{2}}^{*}$

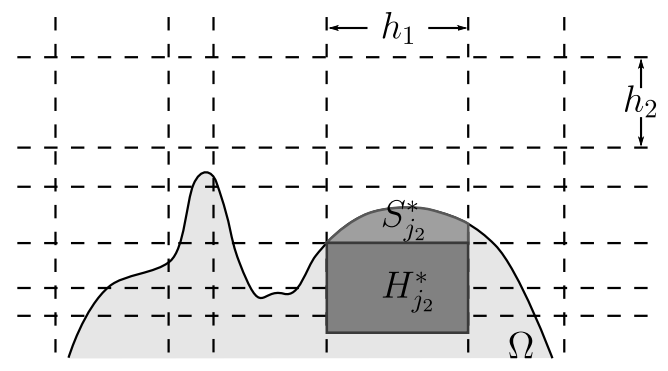

(j) Domain $H_{j_{2}}^{*}$ for local approximation

Figure 1: Examples for diversification, condensation, and related sets 